\title{
BMJ Open Outcomes of specialist discharge coordination and intermediate care schemes for patients who are homeless: analysis protocol for a population-based historical cohort
}

\author{
Ruth M Blackburn, ${ }^{1,2}$ Andrew Hayward, ${ }^{1,2}$ Michelle Cornes, ${ }^{3}$ Martin McKee, ${ }^{4}$ \\ Dan Lewer, ${ }^{1,2}$ Martin Whiteford ${ }^{5}$ Dee Menezes, ${ }^{1,2}$ Serena Luchenski, ${ }^{1,2}$ \\ Alistair Story, ${ }^{6}$ Spiros Denaxas, ${ }^{1,2}$ Michela Tinelli, ${ }^{7}$ Fatima B Wurie, ${ }^{1,2}$ \\ Richard Byng, ${ }^{8}$ Michael C Clark, ${ }^{7}$ James Fuller, ${ }^{3}$ Mark Gabbay, ${ }^{5}$ Nigel Hewett, ${ }^{9}$ \\ Alan Kilmister, ${ }^{3}$ Jill Manthorpe, ${ }^{3}$ Joanne Neale, ${ }^{10}$ Robert W Aldridge $e^{1,2}$
}

To cite: Blackburn RM, Hayward A, Cornes M, et al. Outcomes of specialist discharge coordination and intermediate care schemes for patients who are homeless: analysis protocol for a population-based historical cohort. BMJ Open 2017;7:e019282. doi:10.1136/ bmjopen-2017-019282

- Prepublication history and additional material for this paper are available online. To view these files, please visit the journal online (http://dx.doi. org/10.1136/bmjopen-2017019282).

Received 23 August 2017 Accepted 29 August 2017

CrossMark

For numbered affiliations see end of article.

Correspondence to Dr Robert W Aldridge; R.aldridge@ucl.ac.uk, rob.aldridge@gmail.com

\begin{abstract}
Introduction People who are homeless often experience poor hospital discharge arrangements, reflecting ongoing care and housing needs. Specialist integrated homeless health and care provision (SIHHC) schemes have been developed and implemented to facilitate the safe and timely discharge of homeless patients from hospital. Our study aims to investigate the health outcomes of patients who were homeless and seen by a selection of SIHHC services.
\end{abstract}

Methods and analysis Our study will employ a historical population-based cohort in England. We will examine health outcomes among three groups of adults: (1) homeless patients seen by specialist discharge schemes during their hospital admission; (2) homeless patients not seen by a specialist scheme and (3) admitted patients who live in deprived neighbourhoods and were not recorded as being homeless. Primary outcomes will be: time from discharge to next hospital inpatient admission; time from discharge to next accident and emergency attendance and 28-day emergency readmission. Outcome data will be generated through linkage to hospital admissions data (Hospital Episode Statistics) and mortality data for November 2013 to November 2016. Multivariable regression will be used to model the relationship between the study comparison groups and each of the outcomes. Ethics and dissemination Approval has been obtained from the National Health Service (NHS) Confidentiality Advisory Group (reference 16/CAG/0021) to undertake this work using unconsented identifiable data. Health Research Authority Research Ethics approval (REC 16/EE/0018) has been obtained in addition to local research and development approvals for data collection at NHS sites. We will feedback the results of our study to our advisory group of people who have lived experience of homelessness and seek their suggestions on ways to improve or take this work further for their benefit. We will disseminate our findings to SIHHC schemes through a series of regional workshops.

\section{Strengths and limitations of this study}

- Our study will use data linkage to facilitate largescale evaluation of care for homeless people; a group with significant health needs that is highly mobile and difficult to monitor.

- Data linkage will enable the health of homeless people admitted to hospital to be characterised and changes in their health evaluated with respect to access to specialist integrated homeless health and care schemes.

- Our analysis will be limited to individuals for whom deterministic linkage was possible and restricted to data collected in secondary care settings and mortality.

\section{INTRODUCTION}

Homelessness is associated with high mortality, ${ }^{1} 2$ multiple morbidity and low uptake of preventative interventions. ${ }^{3}$ The most comprehensive assessment to date of the healthcare usage by homeless people in England was published by the Department of Health in March 2010. ${ }^{4}$ This report estimated that healthcare costs ascribed to homeless people were at least $£ 85$ million per year, which is approximately eight times greater-with threefold longer duration of hospital admission-than those of similarly aged adults. ${ }^{4}$

People who are homeless often experience poor hospital discharge arrangements, with the potential to further increase costs through increased rates of readmission. In 2013, the Department of Health launched the 'Homeless Hospital Discharge Fund' (HHDF)—a £10million programme 
allocated to the voluntary sector to develop pilot projects to improve hospital discharge procedures for homeless patients through development of specialist integrated homeless health and care (SIHHC) schemes. ${ }^{5}$ This was partly a response to the finding that $70 \%$ of people who are homeless were being discharged from hospital back to the streets without having their housing or ongoing care needs properly addressed. ${ }^{6}$ In total, 52 discharge coordination and intermediate care 'type' SIHHC schemes were funded through the HHDF. According to an early evaluation report, ${ }^{7}$ the schemes fall into two broad categories:

i. Housing-led schemes: These focus primarily on securing accommodation for people who are homeless on discharge from hospital. Some schemes provide short-term intermediate care follow-up in the community or in a hostel type setting.

ii. Clinically led schemes: These are usually general practitioner or nurse led and involve 'in reach' (hospital ward rounds) and discharge coordination with a focus on both health and housing. These schemes are often referred to as 'pathway discharge teams' in acknowledgement of the Pathway charity that pioneered this way of working. ${ }^{8}$

The clinically led approach has been evaluated in both observational ${ }^{8}$ and interventional ${ }^{9}$ study designs. These analyses found that the duration of hospital admission was not reduced, but quality of life was improved, street homelessness was reduced and patients felt better cared for. Evaluations of schemes using housing support workers tend to be smaller in scale, qualitative and not published in the academic literature.

This study explores the effectiveness of SIHHC as compared with standard care (ie, hospitals which do not have access to a specialist scheme to manage the discharge of patients who are homeless). Our evaluation will work with a sample of 17 SIHHC sites, most of which received funding through the HHDF. We aim to include 7 schemes that are clinically led and 10 that are housing led. Sites will be selected to represent different contexts and localities across England (eg, rural, city, inner London). Our research will be geographically more representative than previous analyses and the use of administrative records may offer a more realistic evaluation of the benefits of the intervention than measurements taken in an interventional study setting. This study contributes to a larger National Institute for Health Research (NIHR)-funded realist evaluation that seeks to understand 'what works' in the delivery of SIHHC. The full protocol for all related work packages is available from the NIHR website. ${ }^{10}$

\section{Methods and analysis}

The study is a historical population-based cohort study. Eligible participants will be adults over 18 years of age with one or more hospital admissions between 1 November 2013 and 30 November 2016.

Individuals will enter the cohort at whichever is the latest of: 1 November 2013; their 18th birthday; hospital admission ('index' admission); SIHHC implementation date. Individuals will be followed up until the earliest of: 30 November 2016, their 100th birthday, death.

For each participant, the index admission will be identified. This is the first admission after the implementation of the SIHHC in the hospital concerned and thus the first at which they could potentially benefit from the scheme, regardless of whether or not they used it. The number of people who died during their index admission will be reported, but these individuals will be excluded and their data will not be included in the analysis beyond this point. Multiple admissions for the same individual less than 1 day apart, including those relating to a hospital transfer, will be assumed to be part of the same admission. ${ }^{11}$

\section{Ethics and information governance}

Health Research Authority Research Ethics Committee approval has been sought and received (REC 16/ $\mathrm{EE} / 0018$ ). In addition, local research and development approvals were set up prior to local data collection at each of the 17 SIHHC sites. To undertake this work, we require access to patient identifiable data without individual consent and have obtained approval (reference 16/Confidentiality Advisory Group (CAG)/0021) from the Secretary of State for Health through the CAG for this work. After data linkage (see Data collection, processing and linkage section), we will destroy all identifying data as shown in figure 1 and undertake all analyses using a deidentified dataset. All study data will be stored on the University College London (UCL) Data Safe Haven, which has been certified to the ISO27001:2013 information security standard and conforms to the National Health Service (NHS) Information Governance Toolkit. ${ }^{12}$

\section{COMPARATOR GROUPS}

Three groups of individuals admitted to hospital will be included in the analysis. The first group comprises homeless individuals admitted to hospital at any one of 17 sites with a SIHHC scheme between 1 November 2013 and 30 November 2016. We have conducted a comprehensive audit of all SIHHC hospital sites as part of the wider NIHR evaluation. Using these data, we have determined the date on which SIHHC schemes were implemented.

The second group comprises individuals seen by a community homeless service in London (Find and Treat $(\mathrm{F} \& \mathrm{~T}))$ and subsequently admitted to a hospital that did not have a SIHHC scheme. For these non-SIHHC hospitals, a hypothetical implementation date will be chosen from within a randomly drawn decile of SIHHC implementation dates. F\&T is a specialist outreach team that works alongside over 200 NHS and third sector front-line services to prevent or treat tuberculosis among homeless people. F\&T is primarily based in London-an issue we will explore further in a sensitivity analysis by restricting the cohort to admissions in London alone. This group is subsequently referred to as F\&T. We will look for hospital admissions during the study period for all individuals 


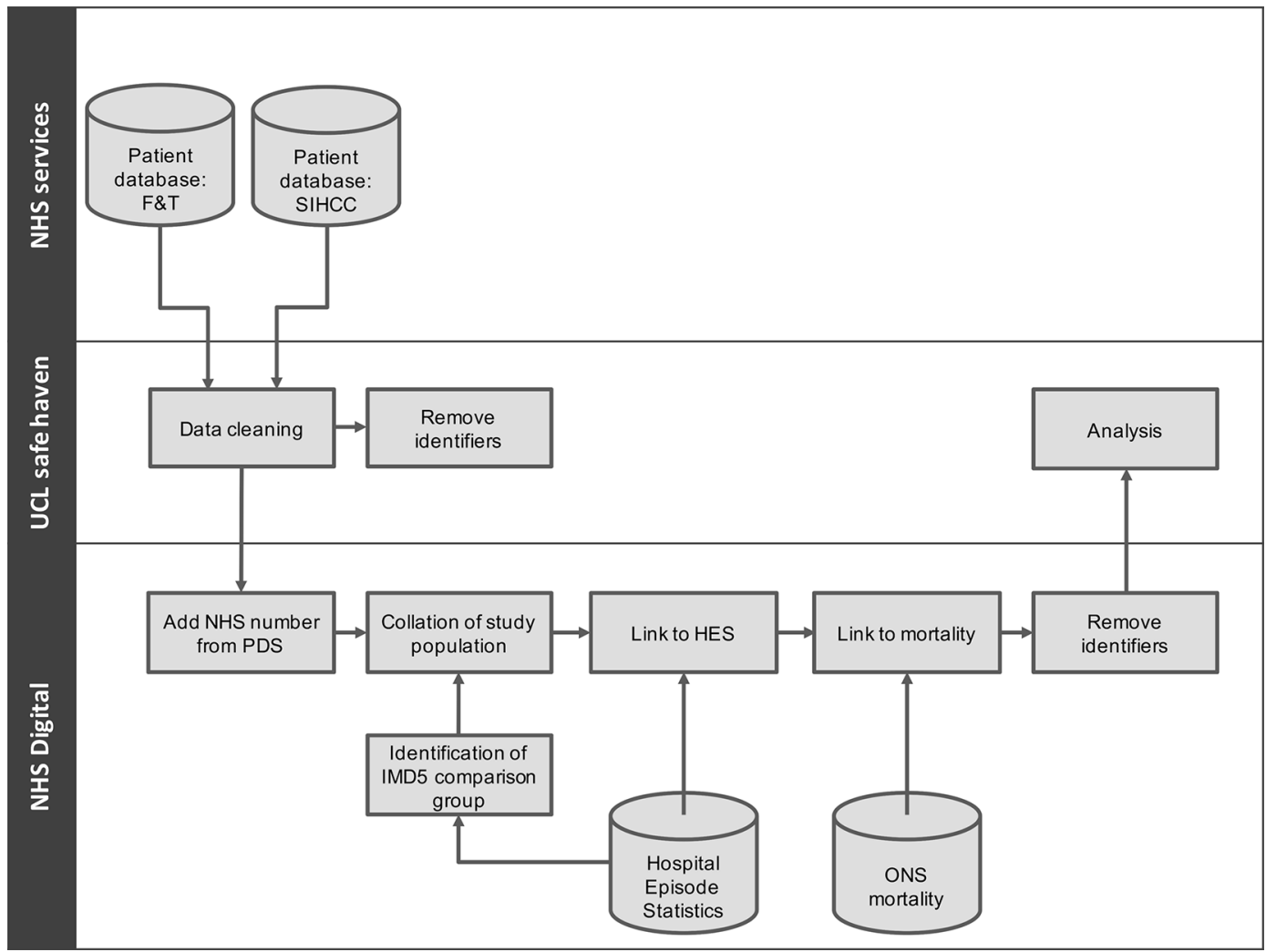

Figure 1 Study data flows. F\&T, Find and Treat; HES, Hospital Episode Statistics; IMD, index of multiple deprivation; NHS, National Health Service; ONS, Office for National Statistics; PDS, Personal Demographics Service; SIHHC, specialist integrated homeless health and care ; UCL, University College London.

seen by F\&T between 1 November 2013 and 30 November 2016.

The third group is a random sample of individuals equal in size to the F\&T group and living in lower super output areas in England in the most deprived quintile, as measured by the index of multiple deprivation (IMD), who are recorded as having a fixed address. These individuals will come from hospitals offering SIHHC schemes with an admission during the study period but without being seen by the SIHHC scheme. This group will be used to confirm that the homeless people included in the study have worse outcomes than non-homeless people living in deprived areas. This group is subsequently referred to as IMD5.

\section{Datasets used in the analysis}

We will draw on three sources of study participants. First, unconsented data collected at SIHHC sites (see ethics and data security section in relation to the approvals and information governance framework applied to carry out this work). This will include identifying demographic data (forename, surname, aliases, date of birth, sex, current or previous postcodes, nationality, ethnicity, NHS number-a unique 10-digit numeric identifier for patients in the healthcare system assigned at first encounter) for relevant hospital inpatients during the study period. Second, unconsented data from F\&T, again including identifying demographic data. Third, hospital records from SIHHC sites for a random sample of anonymous patients from deprived areas will be generated, equal in size to the F\&T group.

Data in respect of each group from Hospital Episode Statistics (HES), which includes dates, causes and length of admission and the Office for National Statistics' mortality database, which includes dates and causes of death, will be linked. In this study, we will be unable to include data from primary care due to the lack of national dataset available for this purpose. As a result, we will be unable to examine any contribution to the health and care of the individuals from primary and community or social care, which is therefore an important limitation of the study.

\section{Data collection, processing and linkage}

Data flows used in this analysis are described in figure 1. We will obtain demographic identifying variables from all individuals at SIHHC sites and F\&T under our legal and ethical approvals. Where available, NHS number will be collected from the 17 SIHHC sites. NHS Digital (formally Health and Social Care Information Centre) will use the Personal Demographics Service (PDS) to identify and add NHS numbers to as many individual records as possible.

NHS Digital will undertake all data linkage to HES data using patient identifiers obtained from the SIHHC sites and F\&T datasets (in combination with PDS tracing) and securely upload a deidentified copy of the data to the University College London Institute of Health Informatics' Data Safe Haven-a robust infrastructure certified 
for processing and analysing identifiable data according to international and national information security standards (ISO/IEC 27001:2013 and NHS Information Governance Toolkit). The deterministic linkage process will be undertaken in three steps to ensure the three groups are mutually exclusive:

Step 1. HES linkage to data collected at the SIHHC sites.

Step 2. HES linkage to data collected from F\&T community-based services, with admissions stratified into those sites with and without SIHHC schemes.

Step 3. Identifying people in the most deprived IMD quintile from HES at the SIHHC sites who were admitted after the implementation date at a given SIHHC site.

In the main analysis, individuals identified in more than one of the steps described above will be classified using the hierarchy $1>2>3$, such that data on homeless patients who were admitted multiple times to a combination of hospitals with, and without, SIHHC schemes will be analysed according to admission to the SIHHC site only. Patients identified as both homeless (ie, SIHHC or F\&T) as well as those in the non-homeless (IMD5) group will be assigned to the homeless category. Sensitivity analyses will examine the impact of excluding people who were identified in more than one group. We will obtain data on all individuals from HES in respect of admissions to any hospital in England from 1 January 2008 to 31 October 2013 to create a profile of their existing health conditions.

\section{SAMPLE SIZE}

Our sample size is based on historical data from the two types of SIHHC scheme, suggesting an average of 92 patients per month across the 17 sites. We therefore estimate that each type will have data from approximately 2208 patients for the duration of the study.

From previous health service evaluations, we expect 0.7 hospital episodes per person-year for homeless individuals. A clinically important reduction in readmission rates would be $10 \%$. To undertake a sample size calculation to determine the study size required to detect such a reduction in readmission rates, the following variables were defined:

$\mu_{-} 0=$ mean readmission rate in the baseline $(\mathrm{F} \& \mathrm{~T})$ group $=0.7$ episodes per person-year.

$\mu_{-} 1=$ mean readmission rate in the SIHHC group $=0.6$ episodes per person-year.

$\mathrm{v}=1.96=$ percentage point of the normal distribution corresponding to a $5 \%$ two-sided significance level.

$\mathrm{u}=0.84=$ one-sided percentage point of the normal distribution corresponding to (100\%- type II error (FN/ $(\mathrm{TP}+\mathrm{FN})))$ at $80 \%$ power.

Using the following equation, we estimated minimum sample size (per group) required for comparison of two rates (readmissions per person-year):

$(\mathrm{u}+\mathrm{v})^{\wedge} 2 *\left(\mu \_1+\mu \_0\right) /\left(\mu \_1-\mu \_0\right)^{\wedge} 2$

$(0.84+1.96) \wedge 2 *(0.7+0.6) /(0.7-0.6) \wedge 2$
$=1019$ person-years per comparator group

Adjustment for confounders (age, sex, ethnicity, calendar quarter of admission and existing comorbidities) as far as is possible within the analysis, results in a doubling of sample size (additional 10\% per confounder). Therefore, 2038 person-years will be required per group. Assuming that the SIHHC sites see on average 90 patients each month during the study period (which for the purposes of the initial calculation we assumed to be November 2013-November 2015 as many schemes may have been shut down before this time period and in some we may collect data at an earlier point), we estimate that this translates to a total 2160 individuals at each of these two types of scheme. Given that the average follow-up period is likely to exceed 1 year, we anticipate that the study will be powered to detect a $10 \%$ difference in readmission rates between SIHHC and non-SIHHC sites, for both the clinically led and the housing-led SIHHC schemes.

\section{OUTCOMES}

A series of primary and secondary outcomes will be included to ensure our analysis is both consistent with outcomes used in previous published analyses and includes outcomes that previous studies were not powered to collect. We have chosen a large number of secondary outcomes that collectively reflect the priorities of health policy-makers and individuals attending our patient engagement workshops. Full definitions of each primary and secondary outcome are provided in tables 1 and 2. In summary they are:

\section{Primary outcomes}

- Time from discharge to next hospital inpatient readmission (any cause)

- Time from discharge to next accident and emergency attendance

- 28-day emergency readmission

\section{Secondary outcomes}

- Time from admission to death due to any cause

- Duration of inpatient admission

- Time from admission to mortality from causes amenable to healthcare ${ }^{13}$

- Time from discharge to admission with ambulatory care sensitive (ACS) conditions

- Time from discharge to next planned admission

- Overall readmission rates

- Unscheduled readmission rates

- All-cause mortality using a standardised mortality ratio (SMR)

- International Classification of Diseases 10th Revision chapter-specific SMR

- In-patient costs using Health Resource Group

\section{ANALYSIS PLAN}

We will undertake the analysis in two phases. In the first phase, we will analyse baseline characteristics (see 
Table 1 Definition and methodological approach for primary outcomes

\begin{tabular}{|c|c|c|}
\hline Primary outcome & Definition & Approach \\
\hline $\begin{array}{l}\text { Time from discharge to next } \\
\text { hospital inpatient admission (any } \\
\text { cause) }\end{array}$ & $\begin{array}{l}\text { Binary indicator for readmission (yes/no). Time to } \\
\text { event defined as index admission discharge date until } \\
\text { the earliest of: } \\
- \text { readmission } \\
- \text { end of follow-up }\end{array}$ & Cox proportional hazards model \\
\hline $\begin{array}{l}\text { Time from discharge to next } A \& E \\
\text { attendance }\end{array}$ & $\begin{array}{l}\text { Binary indicator for subsequent A\&E attendance (yes/ } \\
\text { no). } \\
\text { Time to event defined as index admission discharge } \\
\text { date until the earliest of: } \\
\text { - next A\&E attendance } \\
\text { - end of follow-up }\end{array}$ & \\
\hline 28-day emergency readmission & $\begin{array}{l}\text { Binary indicator for emergency readmission (yes/ } \\
\text { no) recorded within } 28 \text { days of the index admission } \\
\text { discharge date. Emergency admissions are defined as } \\
\text { those where the admission method is } 11,12 \text { or } 13 \text {. }\end{array}$ & Logistic regression \\
\hline
\end{tabular}

A\&E, accident and emergency.

table 3) of all participants to describe the characteristics of each of the study groups at or before the index admission (see figure 2). With the exception of ethnicity, all baseline characteristics are anticipated to be fully observed (chronic disease is presumed to be absent unless recorded). Missing values of ethnicity will be analysed grouped as 'not recorded'.

We will summarise each of the primary and secondary outcomes by comparison group and explore the geographical spread of our 17 sites to explore their representativeness. This work will enable us to confirm the suitability of the proposed statistical methods and analysis protocol proposed. In addition, we will use life tables to estimate the life expectancy of homeless people included within the study, and we will examine overall rates of avoidable deaths and ACS as these have all been poorly estimated within the literature prior to this study.

In the second phase, we will identify evidence of differences in the baseline characteristics including age, sex, chronic disease and reason for hospital admission at the time of their index admission. We will estimate the crude association between each of the primary and secondary outcomes and the study population groups. The baseline comparator group will be F\&T. We will then re-estimate the association between each of the outcomes and the study population group after adjusting for characteristics at the time of admission: age, sex, chronic disease and reason for hospital admission. Finally, we will undertake supplementary subgroup analyses to evaluate evidence of a difference in the outcomes of people admitted to clinically led versus housing-led schemes.

An appropriate statistical model (selected on the basis of meeting assumptions such as proportional hazards for Cox regression) will be used to analyse the relationship between the study comparison group and each of the outcomes outlined in Tables 1,2. Crude models will be fitted prior to adjustment for 'baseline' measurements at or before the index admission. We will write the analysis in accordance with the REporting of studies Conducted using Observational Routinely-collected Data statement. ${ }^{14}$

\section{SENSITIVITY ANALYSES}

There are several challenges when examining differences in the outcomes for users of SIHHC schemes. First, there are likely to be differences in the underlying health risks of the groups and accessibility of services. Second, clinically led schemes may be more likely to collect NHS numbers, potentially improving (and biassing) the accuracy of linkage for this group compared with others. Third, there may be biases between the groups in their propensity to use health services in the immediate period after the study. Those using clinically led schemes may be more likely to return to these services again and be captured more often within HES data than the schemes led by housing support workers. Fourth, it is possible that individuals seen by SIHHC schemes are the most unwell and therefore have poor outcomes regardless of services. Conversely, it is possible (although we think unlikely) that individuals who are homeless compared with those who are housed end up being admitted earlier and have less mortality and morbidity. Our range of primary and secondary outcomes and sensitivity analyses will help us explore these issues further. While we will provide point estimate of effectiveness, more importantly, we believe that our study will produce a plausible range of outcomes for the carefully chosen comparator groups (using confidence and uncertainty intervals).

We will examine these plausible ranges further by conducting the following sensitivity analyses. First, we will repeat analysis only for those individuals for whom it has been possible to generate confounding variables that can be used for adjustment within the statistical models. Second, we will designate a wash out period of 3 months after the index admission date where outcome data for this period are excluded to reduce bias of healthcare 

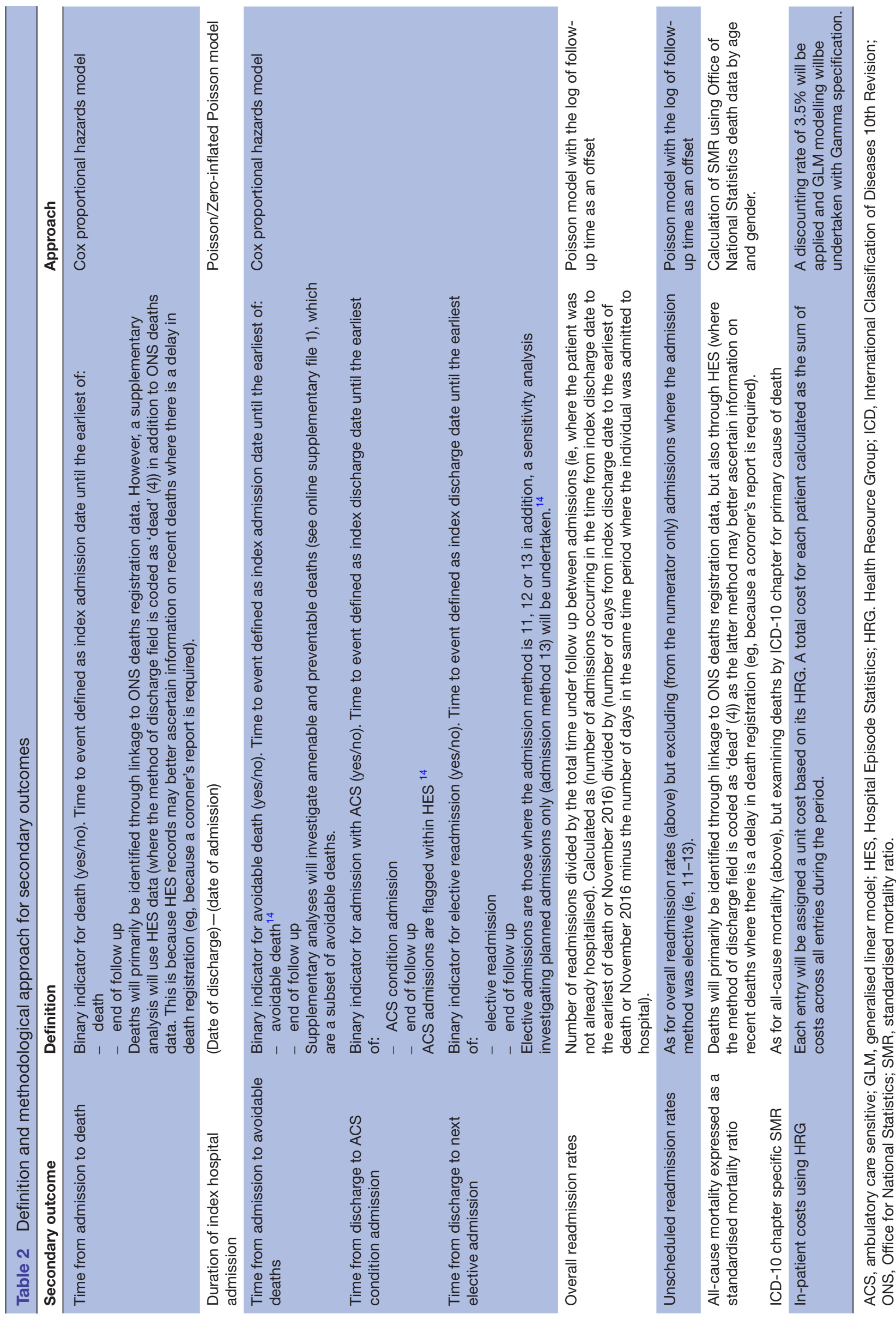
Table 3 Patient characteristics in the time prior to the index admission will be collated as baseline measurements

\begin{tabular}{|c|c|}
\hline Variable & Description \\
\hline Age & $\begin{array}{l}\text { (In years) at a given time point will be } \\
\text { estimated as ((date of admission - } \\
\text { month and year of birth)/365.25) for } \\
\text { the index admission }\end{array}$ \\
\hline Sex & As recorded at the index admission* \\
\hline Ethnicity & As recorded at the index admission* \\
\hline $\begin{array}{l}\text { ICD-10 chronic } \\
\text { disease conditions }\end{array}$ & $\begin{array}{l}\text { Obtained from all admissions at } \\
\text { or before the index admission, } \\
\text { subdivided into categories of: } \\
\text { 1. mental health/behavioural } \\
\text { 2. cancer/blood disorders } \\
\text { 3. chronic infections } \\
\text { 4. respiratory } \\
\text { 5. metabolic/endocrine/nutritional } \\
\text { 6. renal/genitourinary } \\
\text { 7. musculoskeletal/dermatological } \\
\text { 8. neurological } \\
\text { 9. cardiovascular }\end{array}$ \\
\hline Admitting diagnosis & $\begin{array}{l}\text { Reason for index hospital admission } \\
\text { classified according to HRG, which } \\
\text { describes case-mix according to } \\
\text { the chapter and subchapter of the } \\
\text { reason for admission and associated } \\
\text { procedures. }\end{array}$ \\
\hline
\end{tabular}

*Missing information in the index admission record will be completed (where possible) with the modal value from other records for the same individual.

HRG, Health Resource Group; ICD-10, International Classification of Diseases 10th Revision. usage by the initial SIHHC scheme. Third, we investigate the impact of restricting follow-up time to the 6 and 12-month time periods following implementation dates as many of the SIHHC schemes were only operational for 6 to 12 months. Fourth, we will identify deaths using HES data (Method of discharge field, code 4) in addition to deaths identified using Office for National Statistics mortality data. ${ }^{15}$ Fifth, we will conduct separate analyses according to the type of index admission (elective or emergency). Sixth, we will investigate only rates of planned elective readmissions (admission method 13). Seventh, we will restrict our analysis to admissions in London to explore any bias in a comparison with F\&T which undertakes the majority of its screening in community settings in London.

\section{Dissemination and impact}

To help ensure impact from the work, we have engaged with different groups throughout the design and conduct of the study to ensure relevance to their worlds and prepare a pathway for impact and will continue to do this through to the end of the project. In designing this study, we held a workshop with people who had lived experience of homelessness to understand their views on the consent process, data linkage and analysis. At the end of the study, we will reconvene a similar group to feedback the results of our study and seek suggestions on ways to take this work forward. We will also disseminate our findings to SIHHC schemes through a series of regional workshops. 
Author affiliations

${ }^{1}$ Institute of Health Informatics, University College London, London, UK

${ }^{2}$ Farr Institute of Health Informatics Research, University College London, London, UK

${ }^{3}$ Social Care Workforce Research Unit, King's College London, London, UK

${ }^{4}$ Department of Health Services Research \& Policy, London School of Hygiene and Tropical Medicine, London, London, UK

${ }^{5}$ Department of Health Services Research, University of Liverpool, Liverpool, UK

${ }^{6}$ University College London Hospitals, London, UK

${ }^{7}$ Personal Social Services Research Unit (PSSRU), London School of Economics and Political Science, London, UK

${ }^{8}$ Community and Primary Care Research Group, Plymouth University Peninsula

Schools of Medicine and Dentistry, PLYMOUTH, UK

${ }^{9}$ Pathway Charity, London, UK

${ }^{10}$ National Addiction Centre, Institute of Psychiatry, Psychology and Neuroscience, King's College London, London, UK

Contributors RWA, AH, RB, MM and DL proposed the methodological approach for the analysis and MC, MW, DM, SL, AS, SD, MT, FW, RB, MC, JF, MG, NH, AK, JM, JN contributed specific revisions. RWA, RMB and DL drafted the initial protocol and $A H$, MC, MM, MW, DM, SL, AS, SD, MT, FW, RMB, MCC, JF, MG, NH, AK, JM, JN revised and edited the manuscript. MW, JN, MCC and NH contributed detailed contextual information regarding the SIHHC sites, prior evaluations of these services and the involvement of people who had lived experience of homelessness. JF, SD, GM, MG, MM JN, MC, NH and RB focused on ethical approvals and increased the readability of the protocol. RB and DL created the figures and associated text.

Funding This work was supported by the National Institute for Health Research (NIHR), HS\&DR Project number: 13/156/10. We also acknowledge the support from the Farr Institute of Health Informatics Research, funded by The Medical Research Council (MR/K006584/1), in partnership with Arthritis Research UK, the British Heart Foundation, Cancer Research UK, the Economic and Social Research Council, the Engineering and Physical Sciences Research Council, the National Institute of Health Research, the National Institute for Social Care and Health Research (Welsh Assembly Government), the Chief Scientist Office (Scottish Government Health Directorates) and the Wellcome Trust. RWA is supported by an academic clinical lectureship from the National Institute for Health Research (NIHR) and a Wellcome Trust Clinical Research Career Development Fellowship (206602/Z/17/Z). ACH's salary is provided by Central and North West London NHS Community Trust. AS is funded by UCLH Foundation Trust. MG is part funded by the NIHR Collaboration for Leadership in Applied Health Research and Care North West Coast (NIHR CLAHRC NWC). JN is part funded by the NIHR Biomedical Research Centre for Mental Health at South London and Maudsley NHS Foundation Trust and King's College London. The views expressed are those of the author(s) and not necessarily those of the NHS, the Wellcome Trust, the NIHR or the Department of Health, or Public Health England. RB is supported by the NIHR Collaboration for Leadership in Applied Health Research and Care South West Peninsula.

Competing interests $\mathrm{ACH}$ is a trustee of the Pathway: Healthcare for homeless people charity. AS is Clinical Lead and Manager for Find and Treat. NH is Medical Director of Pathway Charity, London, a charity that trains and supports Pathway care coordination teams in hospital.

Patient consent Detail has been removed from this case description/these case descriptions to ensure anonymity. The editors and reviewers have seen the detailed information available and are satisfied that the information backs up the case the authors are making.

Ethics approval Health Research Authority Research Ethics Committee.

Provenance and peer review Not commissioned; peer reviewed for ethical and funding approval prior to submission.
Open Access This is an Open Access article distributed in accordance with the terms of the Creative Commons Attribution (CC BY 4.0) license, which permits others to distribute, remix, adapt and build upon this work, for commercial use, provided the original work is properly cited. See: http://creativecommons.org/ licenses/by/4.0/

(c) Article author(s) (or their employer(s) unless otherwise stated in the text of the article) 2017. All rights reserved. No commercial use is permitted unless otherwise expressly granted.

\section{REFERENCES}

1. Aldridge RWS, Hwang A, Nordentoft SW, et al. The health impact of social exclusion: a systematic review and meta-analysis of morbidity and mortality data from homeless, prison, sex work and substance use disorder populations in high-income countries. Lancet 2017 (in press).

2. Hwang SW. Is homelessness hazardous to your health? Obstacles to the demonstration of a causal relationship. Can J Public Health 2002;93:407-10.

3. Story A, Aldridge RW, Gray T, et al. Influenza vaccination, inverse care and homelessness: cross-sectional survey of eligibility and uptake during the 2011/12 season in London. BMC Public Health 2014;14:44.

4. Office of the Chief Analyst Department of Health. Healthcare for single homeless people. London: Department of Health, 2010.

5. Homeless Hospital Discharge Fund 2013 to 2014- GOV.UK: https:// www.gov.uk/government/publications/homeless-hospital-dischargefund-2013-to-2014 (accessed Aug 2017).

6. Homeless Link and St Mungo's publish report on hospitals and the homeless - GOV.UK. https://www.gov.uk/government/news/ homeless-link-and-st-mungo-s-publish-report-on-hospitals-and-thehomeless (accessed Aug 2017).

7. Investment needed to continue homeless hospital discharge improvement. Homeless Link http://www.homeless.org.uk/connect/ news/2015/feb/09/investment-needed-to-continue-homelesshospital-discharge-improvement (accessed Aug 2017).

8. Hewett N, Halligan A, Boyce T. A general practitioner and nurse led approach to improving hospital care for homeless people. BMJ 2012;345:e5999.

9. Hewett N, Buchman P, Musariri J, et al. Randomised controlled trial of GP-led in-hospital management of homeless people ('Pathway'). Clin Med 2016;16:223-9.

10. Effectiveness and cost-effectiveness of 'usual care' Versus 'specialist integrated care': a comparative study of hospital discharge arrangements for homeless people in England: https://www. journalslibrary.nihr.ac.uk/programmes/hsdr/1315610/\#/ (accessed Aug 2017).

11. Hardelid PD N, Davey J. Development of coding clusters for chronic conditions using data from death certificates linked to hospital admissions. Overview of child deaths in the four UK countries: $\mathrm{RCPCH} 2013$.

12. NHS Information Governance Toolkit. https://www.igt.hscic.gov.uk/ (accessed Aug 2017).

13. Nolte E, McKee M. Does health care save lives? Avoidable mortality revisited. The Nuffield Trust 2004 http://researchonline.lshtm.ac.uk/ id/eprint/15535 (accessed Aug 2017).

14. Benchimol El, Smeeth L, Guttmann A, et al. The REporting of studies Conducted using Observational Routinely-collected health Data (RECORD) statement. PLoS Med 2015;12:e1001885.

15. NHS Digital. HES data dictionary. 2017 http://content.digital.nhs.uk/ hesdatadictionary (accessed Aug 2017).

16. Newton JN, Briggs AD, Murray CJ, et al. Changes in health in England, with analysis by English regions and areas of deprivation, 1990-2013: a systematic analysis for the Global Burden of Disease Study 2013. Lancet 2015;386:2257-74. 